\title{
El envejecimiento, un camino que empezamos a recorrer
}

\author{
Aging, a road we are beginning to travel
}

Dra. Diana Moyao-García*

Citar como: Moyao-García D. El envejecimiento, un camino que empezamos a recorrer. Rev Mex Anestesiol. 2022; 45 (2): 79-81. https://dx.doi.org/10.35366/103880

$\mathrm{L}$ a práctica de la anestesiología incluye el desarrollo de competencias basadas en la apli_cación eficiente de conocimientos médicos actuales, aunada al ejercicio de un juicio médico sólidamente fundamentado que se adapte a diferentes entornos clínicos, además de la capacidad de realizar diferentes tareas cognitivas y técnicas como miembro de un equipo ${ }^{(1)}$. En la medida en que un anestesiólogo no pueda o no esté dispuesto a desempeñarse al más alto nivel en cualquiera de estos ámbitos, se puede considerar que sufre algún grado de deterioro o discapacidad ${ }^{(2)}$.

En la especialidad de anestesiología hay un número cada vez mayor de médicos en ejercicio profesional de 60 años o más. Las razones más comunes entre quienes continúan trabajando después de la edad prevista de jubilación son la satisfacción profesional, las preocupaciones de salud personal y las necesidades financieras. Estos anestesiólogos, de amplia experiencia, son miembros valiosos de cualquier equipo de trabajo; sin embargo, en este contexto, es importante no perder de vista los cambios fisiológicos relacionados con la edad, que aparecen en diferente grado en todos los individuos y que pueden afectar la práctica profesional del anestesiólogo mayor ${ }^{(3)}$.

El envejecimiento está asociado con una serie de cambios fisiológicos y cognitivos que pueden disminuir la capacidad de un anestesiólogo para continuar brindando una atención anestésica segura y eficiente. Una evaluación frecuente de sus habilidades clínicas y la conciencia personal de sus limitaciones, pueden hacer posible que un anestesiólogo que envejece continúe ejerciendo una práctica exitosa ${ }^{(4)}$.

En términos generales, el envejecimiento puede definirse como un deterioro gradual de la función fisiológica, los cambios físicos y funcionales van acompañados también de cambios psicológicos, espirituales y sociales. El proceso de envejecimiento está asociado con cambios degenerativos en la estructura y función de las células y tejidos. Cada sistema envejece a su propio ritmo y, en consecuencia, puede haber grandes diferencias en las capacidades funcionales de diferentes órganos; no obstante, un factor común en todo el organismo es una disminución de las reservas fisiológicas. Una parte de estas reservas se emplean para mantener la homeostasis, dejando una capacidad limitada para compensar necesidades adicionales, esto hace que el organismo que envejece sea cada vez más vulnerable a todas las formas de enfermedad ${ }^{(3)}$.

El envejecimiento no es una discapacidad; sin embargo, muchos de los cambios fisiológicos asociados provocan deficiencias específicas que pueden interferir con la capacidad de un anestesiólogo para un ejercicio profesional seguro. Entre las alteraciones relacionadas con la edad que pueden tener un impacto potencial en el rendimiento del anestesiólogo se encuentran las siguientes:

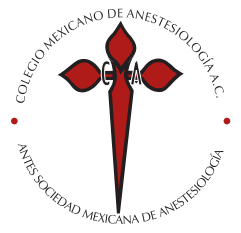

* Anestesióloga Pediatra y Algóloga. Subdirección de Asistencia Quirúrgica. Hospital Infantil de México «Federico Gómez».

Correspondencia:

Diana Moyao-García Dr. Márquez 162,

Col. Doctores, C.P. 06720

Alcaldía Cuauhtémoc,

CDMX. México.

E-mail: dianitamoyao@gmail.com 
1. Los anestesiólogos parecen ser particularmente vulnerables a la pérdida de audición (común en personas mayores) en el rango de alta frecuencia, que puede ser crítica, pues es el rango auditivo de muchas alertas y alarmas del equipo anestésico. Cerca de $40 \%$ de los anestesiólogos mayores de 65 años no pueden escuchar una o más de las alarmas integradas en el equipo estándar. El ruido ambiental excesivo que se experimenta en los quirófanos en la actualidad, se suma a la dificultad que tiene el anestesiólogo mayor para escuchar conversaciones vitales y alarmas ${ }^{(3)}$.

2. Las deficiencias visuales relacionadas con la edad (presbicia, cataratas y otras), causan una disminución de la agudeza visual, la percepción de profundidad y la discriminación del color. Estas alteraciones hacen más difícil realizar tareas visuales en las diferentes condiciones de luz ambiental que se dan en los quirófanos. Hay tareas visualmente complejas (ej. ecografía) que pueden ser especialmente problemáticas en caso de intentar aprender nuevas habilidades ${ }^{(2)}$.

3. Existe una disminución de la masa muscular con la consiguiente pérdida de destreza manual, fuerza y resistencia. $\mathrm{Al}$ anestesiólogo mayor le puede resultar más difícil tolerar períodos de trabajo prolongados y guardias nocturnas, haciéndolo más susceptibles a errores; estas dos situaciones se encuentran entre los aspectos más estresantes de la práctica y las razones más importantes para la jubilación ${ }^{(2)}$.

4. Otras funciones sujetas al deterioro del envejecimiento son los movimientos finos, la percepción táctil y la planificación del movimiento. Con la edad, los individuos muestran alteraciones de la motricidad fina y una reducción de la fluidez del plan de movimiento; éste se manifiesta como un deterioro progresivo con dificultades para iniciar, realizar y controlar el movimiento. Algunos de estos cambios empiezan a producirse a partir de los 45 años y se acentúan significativamente a partir de los 60 , con un declive más pronunciado después de los 75 años. Sin embargo, factores como el entrenamiento, la motivación y el estilo de vida influyen en la preservación de las funciones músculoesqueléticas a lo largo del tiempo ${ }^{(5)}$.

5. Con la edad, las interrupciones del sueño son más frecuentes y las fases de sueño reparador disminuyen; esto se traduce en un estado crónico de fatiga, que a su vez puede conducir a un deterioro de la función cognitiva percibido principalmente como alteraciones en la atención. Realizar guardias nocturnas puede ser muy perjudicial para el patrón de sueño, así mismo, la fatiga tiene un efecto deletéreo en el desempeño y estado de ánimo de los médicos mayores ${ }^{(5)}$.

6. El desafío más amenazante para una práctica anestésica segura lo representa el deterioro cognitivo. Hay cambios anatómicos y funcionales en el sistema nervioso central, que se presentan entre los individuos que envejecen. Se alteran muchos aspectos de la memoria, la abstracción y la flexibilidad mental; la memoria a corto plazo se ve afectada, la capacidad de atención disminuye, y el aprendizaje se vuelve más lento y requiere más esfuerzo. Además, también hay un deterioro en el pensamiento creativo, la capacidad de resolución de problemas y el razonamiento; así como disminución en la rapidez intelectual y lentificación del tiempo de reacción. Los cambios en la cognición pueden verse exacerbados por muchas de las enfermedades asociadas con la vejez, como la diabetes, los accidentes cerebrovasculares, las enfermedades cardiovasculares y las enfermedades infecciosas ${ }^{(3)}$.

En contraparte, hay aspectos importantes de la cognición que con frecuencia no se ven afectados durante el envejecimiento saludable; la memoria a largo plazo suele permanecer intacta aun frente a una pérdida marcada de la memoria a corto plazo. Rasgos de personalidad como el optimismo, la resiliencia y la compasión suelen permanecer intactos; habilidades críticas para un anestesiólogo como la sabiduría y el juicio frecuentemente mejoran con la edad ${ }^{(3)}$. La mayoría de los anestesiólogos mayores que gozan de buena salud continúan desempeñándose profesionalmente bien; aunque pueden ser más lentos para reconocer y manejar situaciones nuevas, responden con la misma rapidez cuando no están cansados y pueden aprovechar su valiosa experiencia ${ }^{(4)}$.

En situaciones clínicas que evolucionan rápidamente, los anestesiólogos mayores se basan en la experiencia previa, reconocen de manera intuitiva los patrones haciendo que la «rutina» sea automática y la respuesta rápida, se basan más en el reconocimiento de patrones que en el análisis. En una situación que ameritara una solución urgente, esto podría ser una ventaja y se podría "ganar tiempo», en comparación con una conducta de análisis y razonamiento conscientes, propia de un profesional joven. El problema potencial surge cuando el anestesiólogo mayor no se da cuenta de que una situación está cambiando, malinterpreta los eventos o no tiene experiencia previa en la cual basarse. Los anestesiólogos mayores generalmente reciben menos retroalimentación sobre su desempeño y pueden tener más dificultades para reconocer cuando sus habilidades se deterioran ${ }^{(4)}$.

Aunque es difícil identificar una asociación directa entre las deficiencias específicas del envejecimiento, las habilidades clínicas y la competencia de un médico, diferentes estudios han encontrado a la edad avanzada como un predictor de bajo rendimiento en pruebas estandarizadas de competencia profesional. Hay publicaciones que brindan información sobre el desempeño de los anestesiólogos mayores; en un estudio diseñado para determinar la competencia en un escenario simulado de «no poder intubar/no poder ventilar» con cricotiroidotomía de emergencia simulada, reportó que el aumento de la edad del operador y los años desde el egreso de la residencia se asociaron con una disminución de la competencia. Un informe 
canadiense sobre las denuncias presentadas contra anestesiólogos demostró en los mayores de 65 años un riesgo 1.5 veces mayor de ser encontrado responsable, en comparación con los de menos de 51 años; además, la gravedad de las lesiones fue dos veces mayor entre los anestesiólogos de más edad ${ }^{(2)}$.

Hay excepciones sobre la observación generalizada de un rendimiento disminuido en los exámenes estandarizados entre los médicos que envejecen, porque existe un amplio espectro de rendimiento en muchos de estos exámenes entre los médicos mayores. Sin embargo, uno de los hallazgos más sólidos en la investigación sobre el envejecimiento es que la variabilidad entre las puntuaciones que reciben las personas tiende a aumentar con la edad ${ }^{(5)}$.

Algunas publicaciones han enfatizado la necesidad crucial de capacitación para mantener las habilidades técnicas de los anestesiólogos que envejecen; una forma de minimizar la tendencia hacia la disminución del rendimiento es mediante la participación reiterada en programas de educación médica continua de «alta calidad».

Para superar estas deficiencias, se ha evidenciado que la simulación de alta fidelidad es un método apropiado para la evaluación exhaustiva y objetiva de las competencias de los médicos ${ }^{(1)}$. La simulación proporciona un medio para detectar y tal vez revertir la disminución de la capacidad asociada con la edad. Una evaluación de competencias en sesiones de simulación podría desempeñar un papel crucial en el mantenimiento de un alto nivel de atención y un nivel adecuado de seguridad del paciente; con la posibilidad de ofrecer programas de recuperación específicos para la capacitación a través de la simulación, si se detectara una disminución sustancial de la competencia ${ }^{(5)}$.

Publicaciones recientes indican que la simulación de alta fidelidad permite que la experiencia clínica y las habilidades psicomotoras adquiridas en el quirófano puedan evaluarse de manera objetiva y confiable. La simulación de alta fidelidad reproduce condiciones psicológicas relacionadas con el manejo de procedimientos complejos «salva vidas», que contribuyan a mejorar el rendimiento y el mantenimiento de competencias a lo largo del tiempo. Lo más importante es que se ha demostrado que la competencia adquirida durante la simulación se transfiere a la práctica clínica, tanto en la Terapia Intensiva como en quirófanos ${ }^{(5)}$.

La simulación permite a los anestesiólogos mayores evaluar su práctica y también ha demostrado ser útil para detectar los factores responsables del bajo rendimiento asociado con el envejecimiento y corregirlos. Se ha identificado a los escenarios de simulación de alta fidelidad como una de las herramientas que pueden resultar útiles para evaluar y mejorar la competencia de una fuerza laboral que envejece ${ }^{(5)}$.

\section{REFERENCIAS}

1. Tetzlaff JE, Warltier DC. Assessment of competency in anesthesiology. Anesthesiology. 2007;106:812-825.

2. Katz JD. The impaired and/or disabled anesthesiologist. Curr Opin Anesthesiol. 2017;30:217-222.

3. Katz JD. The aging anesthesiologist. Curr Opin Anesthesiol. 2016;29:206-211.
4. Redfern N, Clyburn P, Grigoras I, Calleja P, Mellin-Olsen J, NovakJankovic V, Bobek I, De Hert S, Zacharowski K, De Robertis E. Getting old. Eur J Anaesthesiol. 2020;37:1-3.

5. Giacalone M, Zaouter C, Mion S, Hemmerling TM. Impact of age on anaesthesiologists' competence. A narrative review. Eur J Anaesthesiol. 2016;33:787-793. 